\section{Gambling Rats: Insight into Impulsive and Addictive Behavior}

Although nothing more than a harmless recreation for most, gambling can become a 'behavioural addiction' comparable to drug dependency (Potenza, 2008). The incidence of such pathological gambling (PG) in the USA is estimated at $1-5 \%$, yet current treatments are limited and often ineffective. Animal models of substance use have made a significant contribution to our understanding of drug addiction. Modeling gambling processes in non-human subjects could likewise facilitate our understanding of the drive to gamble, and stimulate the development of pharmacotherapies.

Clinically, one of the most common tests of gambling-related decisionmaking is the Iowa Gambling Task (IGT), in which subjects choose from four decks of cards to win money or points (Bechara et al, 1994). Two of the decks are associated with bigger immediate rewards but disproportionately large losses. The correct approach is therefore to favour decks which deliver smaller amounts but also lower penalties, thereby leading to greater long-term gain. Using the IGT as a template, novel rodent models of gambling behaviour have been developed in which rats play against the clock to earn as many sugar pellets as possible by sampling between four different options. The amount of reward available on each option varies in size (1-4 pellets). On a given trial, the animal receives either the set reward or a punishing 'time-out' during which reward cannot be earned. The reinforcement schedules are fixed such that the larger pellet options are associated with a greater frequency or duration of time-outs, decreasing their net worth. Rats must therefore learn to avoid risky options associated with larger rewards, analogous to the optimal strategy on the IGT. Recent data indicate that rats are capable of 'playing the odds' in this way. Furthermore, such choice behaviour can be modulated by drugs which target the serotonin and dopamine systems, with a $\mathrm{D}_{2}$ receptor antagonist enhancing performance, while amphetamine and a serotonin $1 \mathrm{~A}$ receptor agonist impair choice (Zeeb et al, 2009). Animals which prefer the maladaptive 'high-risk high-reward' options also show elevated preference for exposed or novel environments, indicating this choice pattern may be a valid marker for risk-prone behaviour (Rivalan et al, 2009).

Human and animal studies indicate that highly impulsive individuals, particularly those showing elevated levels of motor impulsivity, are more vulnerable to both cocaine dependency and PG (Verdejo-Garcia et al, 2008). Whether this form of impulsivity represents an endophenotype for chemical and behavioural addictions remains an interesting hypothesis now open to empirical verification. Demonstrating that rodents can engage in a decision-making process reminiscent of gambling, and that such decision-making can be pharmacologically modified, is certainly a step forward. As with research into substance abuse, however, it will be important to dissociate the act of performing a potentially-addictive behaviour and actually exhibiting symptoms of dependence. Some of the DSM-IV criteria for drug dependency have been translated into behavioural measurements applied to rats selfadministering cocaine, theoretically demarcating dependent subjects (Belin et al, 2008). A future challenge for the field will be to create a similar standard for gambling models to help identify subjects exhibiting a PG-like phenotype.

\section{Catharine A Winstanley ${ }^{1}$}

${ }^{1}$ Department of Psychology, University of British Columbia, Vancouver, BC, Canada

E-mail: cwinstanley@psych.ubc.ca

\section{DISCLOSURE}

The author declares no conflict of interest.

Bechara A, Damasio AR, Damasio H, Anderson SW (1994). Insensitivity to future consequences follow- ing damage to human prefrontal cortex. Cognition 50: 7-15.

Belin D, Mar AC, Dalley JW, Robbins TW, Everitt BJ (2008). High impulsivity predicts the switch to compulsive cocaine-taking. Science 320 : 1352-1355.

Potenza MN (2008). Review. The neurobiology of pathological gambling and drug addiction: an overview and new findings. Philos Trans $R$ Soc Lond B Biol Sci 363: 3181-3189.

Rivalan M, Ahmed SH, Dellu-Hagedorn F (2009). Risk-prone individuals prefer the wrong options on a rat version of the lowa Gambling Task. Biol Psychiatry 66: 743-749.

Verdejo-Garcia A, Lawrence AJ, Clark L (2008). Impulsivity as a vulnerability marker for substanceuse disorders: review of findings from high-risk research, problem gamblers and genetic association studies. Neurosci Biobehav Rev 32: 777-810.

Zeeb FD, Robbins TW, Winstanley CA (2009). Serotonergic and dopaminergic modulation of gambling behavior as assessed using a novel rat gambling task. Neuropsychopharmacology 34: 2329-2343.

Neuropsychopharmacology Reviews (2011) 36, 359; doi: 10.1038/npp.2010.136

\section{Insulin Regulation of Monoamine Signaling: Pathway to Obesity}

The prevalence of obesity and related disorders such as diabetes has skyrocketed worldwide despite efforts to therapeutically target homeostatic mechanisms that regulate appetite, energy expenditure, and weight gain. The failure of these efforts points to the existence of additional, nonhomeostatic mechanisms that mediate feeding behavior (Palmiter, 2007). Indeed, redundancy in these systems makes obesity therapy difficult as further evidenced by the failure of newer drugs targeting distinct aspects of these systems. Thus, the epidemic of obesity begs for novel concepts and therapeutic targets that ideally treat 'food-use' disorders and related comorbidities such as drug addiction and neuropsychiatric disorders.

Nonhomeostatic or 'reward' circuits originating in dopamine-rich brain structures, which provide motivation and reward stimuli for feeding, are increasingly understood at the cellular and molecular levels (Palmiter, 2007). Long recognized as an important mediator of feeding behavior, dopamine 